ESJ Humanities

\title{
Riziculture pluviale de bas-fonds dans la région de Sédhiou (Sénégal) : contraintes de production et stratégie d'adaptation
}

\author{
Sécou Omar Diedhiou
}

Docteur en Géographie, chercheur associé à l'UMR CNRS 6590 ESO-

Nantes et au Laboratoire de Géomatique et d'Environnement,

Ziguinchor, Sénégal

Mamadou Thior

Docteur en Géographie, chercheur associé au Laboratoire de Géomatique et d'Environnement, Ziguinchor, Sénégal

\section{Adama Cheikh Diouf}

Docteur en Géographie, chercheur associé au Laboratoire : Leïdi,

Université Gaston BERGER de Saint-Louis du Sénégal

\section{Issa Mballo}

Docteur en Géographie, chercheur associé au Laboratoire de Géomatique et d'Environnement, Ziguinchor, Sénégal

\section{Ansoumana Kouma Diallo}

Doctorant en Géographie à l'Université Cheikh Anta Diop de Dakar (UCAD), Laboratoire : Groupe de recherche et d'Appui au développement

(GERAD)

\section{Doi:10.19044/esj.2021.v17n24p88}

Submitted: 04 March 2021

Accepted: 02 July 2021

Published: 31 July 2021
Copyright 2021 Author(s)

Under Creative Commons BY-NC-ND

4.0 OPEN ACCESS

Cite As:

Diedhiou S.O., Thior M., Diouf A.C., Mballo I. \& Diallo A.K. (2021). Riziculture pluviale de bas-fonds dans la région de Sédhiou (Sénégal) : contraintes de production et stratégie d'adaptation. European Scientific Journal, ESJ, 17(24), 88.

https://doi.org/10.19044/esj.2021.v17n24p88

\section{Résumé}

La vallée de Badobar, polarisant 23 villages, est située à cheval entre les communes de Mangaroungou Santo et de Simbandi Brassou. Elle présente un potentiel de terres rizicultivables d'environ 1500 ha. Cette riziculture est globalement pluviale, car elle se produit uniquement pendant l'hivernage. Cependant, la dépendance à la pluviométrie fait que les productrices sont confrontées à des contraintes sociales et environnementales qui amenuisent la productivité du riz. Le but du présent article est d'analyser les contraintes à la 
production rizicole de bas-fonds et d'identifier les stratégies d'adaptation. La méthodologie adoptée s'appuie sur l'exploitation de données quantitatives à partir d'une population cible de 13333 habitants. Au total, 8 villages représentatifs des vallées rizicoles ont été retenus pour les enquêtes de terrain. La démarche qualitative s'est aussi appuyée sur le recueil de récits de vie de 10 agricultrices de la vallée. Les résultats révèlent que la riziculture de basfonds fait face à des problèmes (climatiques) qui ralentissent son développement. La problématique la plus récurrente évoquée par les rizicultrices est la salinisation (61\%). L'ensablement (30\%) et l'acidification (9\%) représentent aussi un problème central. Par ailleurs, des facteurs socioéconomiques bloquent le développement de la riziculture dans la vallée (95\% des femmes n'ont pas de sources de financement et 55\% n'arrivent pas à se procurer ni les fertilisants ni les produits phytosanitaires). Face à ces difficultés, les paysannes mettent en œuvre des stratégies durables d'adaptations (nouvelles variétés de riz) qui participent à l'amélioration des rendements (700 à $800 \mathrm{~kg}$ de riz paddy dans une parcelle de $500 \mathrm{~m}^{2}$ ), à la conservation et la valorisation des ressources naturelles (eau, terre). Ainsi, $66 \%$ des rizicultrices ont recours à une nouvelle variété de riz.

Mots clés : Riziculture pluviale, bas-fonds, contraintes de production, stratégies d'adaptation, Sédhiou, vallée de Badobar (Sénégal) 


\title{
Low-Lying Rainfed Rice Growing in the Sédhiou Region (Senegal): Production Constraints and Adaptation Strategy
}

\section{Sécou Omar Diedhiou}

Docteur en Géographie, chercheur associé à l'UMR CNRS 6590 ESO-

Nantes et au Laboratoire de Géomatique et d'Environnement, Ziguinchor, Sénégal

\section{Mamadou Thior}

Docteur en Géographie, chercheur associé au Laboratoire de Géomatique et d'Environnement, Ziguinchor, Sénégal

\section{Adama Cheikh Diouf}

Docteur en Géographie, chercheur associé au Laboratoire : Leïdi, Université Gaston BERGER de Saint-Louis du Sénégal

\section{Issa Mballo}

Docteur en Géographie, chercheur associé au Laboratoire de Géomatique et d'Environnement, Ziguinchor, Sénégal

\section{Ansoumana Kouma Diallo}

Doctorant en Géographie à l'Université Cheikh Anta Diop de Dakar (UCAD), Laboratoire : Groupe de recherche et d'Appui au développement

(GERAD)

\begin{abstract}
The Badobar valley, polarizing 23 villages, is located between the communes of Mangaroungou Santo and Simbandi Brassou. It has a potential for rice cultivation of around 1,500 ha. This rice cultivation is generally rainfed there, as it occurs only during the rainy season. However, the dependence on rainfall means that women producers are confronted with social and environmental constraints that reduce productivity. The aim of this article is to analyze the obstacles to lowland rice production and to identify adaptation strategies. The methodology adopted is based on the use of quantitative data from a target population of 13,333 . In total, 8 villages representative of rice-growing valleys were selected for the field surveys. The qualitative approach was also based on the collection of life stories from 10 women farmers in the valley. The results reveal that lowland rice cultivation faces (climatic) problems which slow down its development. The most recurring problem mentioned by women rice farmers is salinization $(61 \%)$. Siltation (30\%) and acidification (9\%) are also a central problem. In addition, socioeconomic factors are blocking the development of rice cultivation in the valley (95\% of women have no sources of finance and 55\% cannot get hold of fertilizers such as phytosanitary products). Thus, faced with these difficulties, the peasants implement sustainable adaptation strategies (new varieties of
\end{abstract}


rice) which help to improve yields (700 to $800 \mathrm{~kg}$ of paddy rice in a plot of $500 \mathrm{~m}^{2}$ ), conservation and development of natural resources (water, land). Thus, $66 \%$ of women rice farmers use a new variety of rice.

Keywords: Rainfed rice production, lowlands, production constraints, adaptation strategies, Badobar Valley (Senegal)

\section{Introduction}

Au Sénégal, le riz pluvial représente entre 50 et $55 \%$ de la production de riz (ANSD, 2020). Cependant, les rendements restent assez faibles et ne dépassent pas les 1,5 tonne par hectare sur un potentiel de 3 à 3,5 t/ha (Mendez, 2019 ; Mendez et al., 2013). Le riz pluvial couvre moins de 50\% des besoins alimentaires des producteurs et de leurs familles (République du Sénégal, 2014). Aussi, la riziculture pluviale est-elle pratiquée essentiellement dans la région Sud du pays en Casamance et dans une moindre mesure dans la région du Bassin arachidier au Centre du pays notamment Kaolack, Kaffrine et Fatick. La région de Sédhiou dispose d'un potentiel de 56000 hectares de terres en riziculture pluviale dont 36000 ha dans différentes vallées, et 20000 ha sur le plateau (Mendez et al., 2019). Dans cette région, la riziculture se pratique généralement à une petite échelle le long des vallées inondables et encore de manière assez traditionnelle (Mendez et al., 2019). C'est une riziculture de type familial dominée par les femmes avec des outils aratoires rudimentaires (Diédhiou et al., 2018).

Par ailleurs, depuis près de trois décennies, la riziculture pluviale dans la région de Sédhiou est tributaire des aléas des changements climatiques notamment la modification du régime des précipitations, la salinisation, l'acidification et l'ensablement des rizières etc. (Diallo, 2014). D'ailleurs, les populations, particulièrement celles du monde rural, sont le plus exposées aux impacts de la variabilité climatique à cause de leurs systèmes de production ou de cultures moins performantes et étroitement dépendantes de la pluviométrie (Sy \& Mballo, 2017 ; Thior et al., 2019). Ces facteurs d'ordre naturel entraînent des perturbations agricoles brutales qui ont des répercussions profondes sur la vie des populations en général et des agriculteurs en particulier (Thior et al., op.cit). En effet, la riziculture qui assurait l'autonomie alimentaire de la population rencontre des difficultés liées à la faiblesse des rendements et à la réduction des superficies emblavées annuellement.

Cette réduction des superficies emblavées est causée par un manque de main-d'œuvre, parce que le renouvellement de celle-ci pose un réel problème et est exacerbé par l'émigration de jeunes vers les villes (Mendy, 2018). Les jeunes filles (élèves et étudiantes) préfèrent passer leurs vacances dans les grandes villes comme Dakar ou Ziguinchor. Ainsi, ne s'activent-elle 
pas dans les rizières que des femmes d'une tranche d'âge de plus de 40 ans (Mballo et al., 2019; Sané, 2017). Dans la région, durant les deux dernières décennies, les campagnes ont perdu une partie de leur population au profit des villes à la recherche de meilleures conditions de vie, ce qui entraîne une fuite de la main-d'œuvre à la suite de l'exode rural (Mendez, 2013). Les variétés traditionnellement cultivées arrivent difficilement à maturation à cause de l'assèchement précoce des rizières de plus en plus ensablées (Faye et al., 2018 ; Mendy, 2018). De plus, les enjeux des changements climatiques constituent des facteurs aggravant leur impact socio-économique sur les ménages (Sané et al., 2015). Dans un tel contexte, les populations notamment les riziculteurs mettent en place des stratégies d'adaptation pour améliorer les conditions de pratique de la riziculture pluviale. Ces actions consistent à la construction de digues de rétention d'eau et d'anti-sels, de fosses de compostages et la participation à la formation et à l'encadrement des paysans etc.

Comprendre les dynamiques actuelles et les enjeux de la riziculture pluviale dans la région de Sédhiou au Sénégal en termes de contraintes de production et de stratégie d'adaptation est ce qui a motivé le présent travail. Plus spécifiquement, il s'agit d'analyser les contraintes climatiques défavorables à la riziculture pluviale dans la vallée de Badobar, mais aussi l'apport des stratégies individuelles, traditionnelles et collectives déployées par les acteurs locaux pour le maintien de la riziculture pluviale dans ce terroir.

\section{Matériels et méthode}

La méthodologie est basée sur des enquêtes et des observations sur le terrain. La première est quantitative et est axée sur l'administration de questionnaire. La seconde est de type qualitatif ; elle renvoie à des entretiens directifs et semi-directifs (agricultrices, ONG, élus locaux, Organisation Paysanne). La démarche qualitative s'est aussi appuyée sur le recueil de récits de vie de 10 agricultrices de la vallée de Badobar. Le traitement et l'analyse des données statistiques ont permis d'étudier les contraintes de production de la riziculture pluviale de bas-fond dans la zone de Sédhiou. Le questionnaire a porté sur: (1) les pratiques de la riziculture, (2) les contraintes liées à la production et (3) les stratégies développées par les productrices. Par ailleurs, les entretiens ont permis d'analyser en profondeur la problématique de la production rizicole dans les 23 villages que regroupe la vallée de Badobar. 


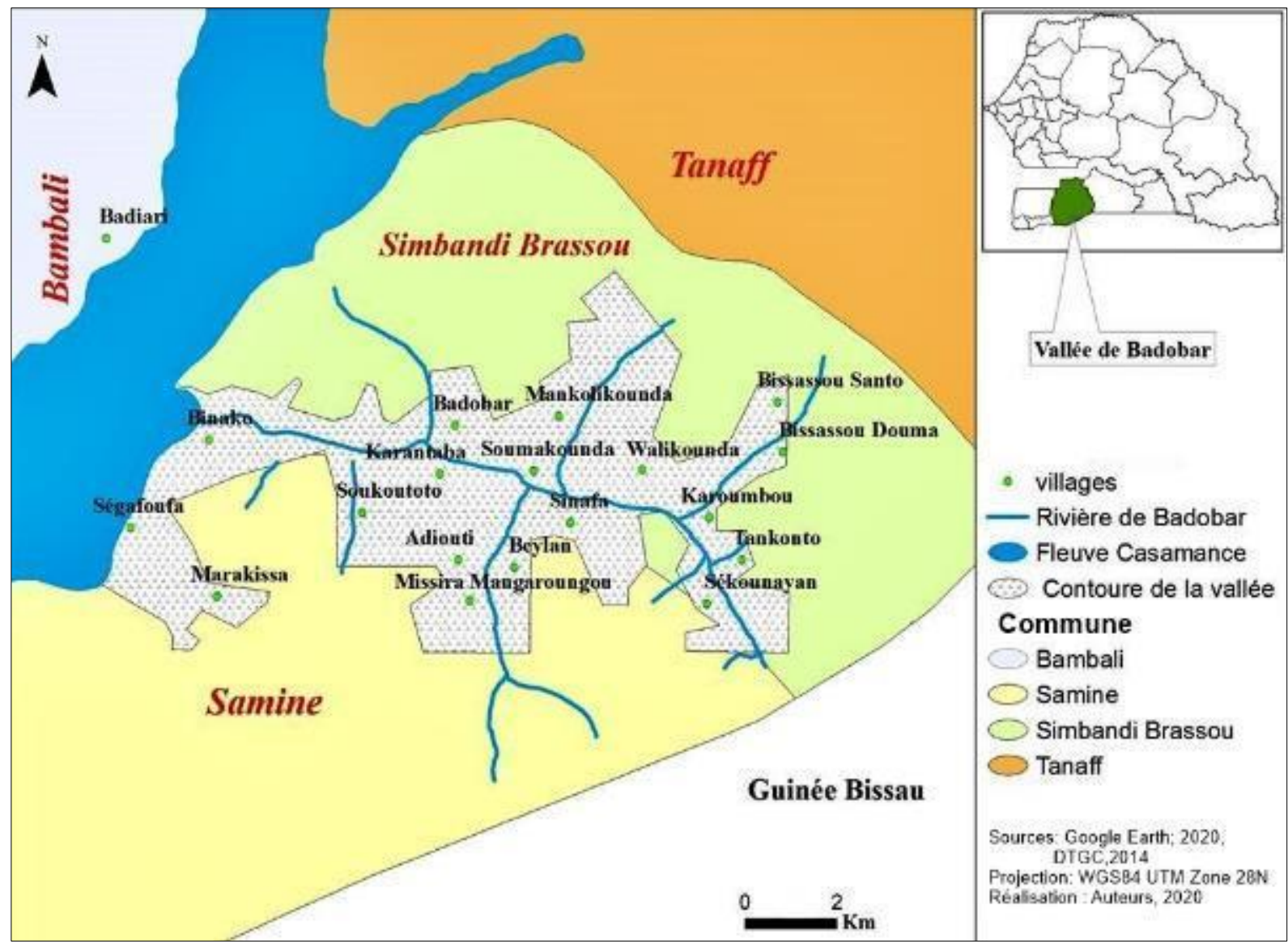

Figure 1 : Situation géographique de la vallée de Badobar

Ce choix a tenu compte des productrices par rapport à la localisation des exploitations où les pratiques rizicoles sont relativement importantes. En effet, c'est à l'échelle des villages choisis qu'on observe fondamentalement les changements environnementaux et socioéconomiques les plus déterminants et plus ou moins homogènes. En outre, la représentativité ethnique ou culturelle, la proximité de la vallée des lieux d'habitation, la présence d'ouvrages hydroagricoles et le poids démographique sont autant d'éléments qui ont été déterminants dans le choix des ménages à interroger. L'unité déclarante est le ménage et l'unité de référence est la productrice de riz (Mendy \& Sy, 2015). De ce fait, il a été appliqué la méthode de détermination suivante par village : soit $N$ le nombre total de ménages recensés par zone et $\boldsymbol{n}$ le nombre de productrices enquêtées. Toutefois, il a été pris en considération le taux de sondage aléatoire selon les estimations statistiques par un pourcentage d'erreur par zone.

La technique utilisée est l'échantillonnage par quota. Dans le cadre de cette recherche, un taux de sondage régulier de $10 \%$ a été opéré sur les 
ménages. De ce fait, en utilisant les données sur le nombre de ménages, ont été interrogées 422 productrices de riz.

En parallèle, l'analyse des données pluviométriques à la station de Sédhiou a permis de déceler des effets complexes de l'empreinte climatique sur la baisse des rendements rizicoles puisque cette dernière est pluviale.

2. Résultats : une diversité de déterminants d'ordre naturel et anthropique influence la déprise de la riziculture pluviale dans la vallée de Badobar

Dans les lignes suivantes, seront présentés les facteurs d'ordre naturel et anthropique qui entraînent la diminution des rendements rizicoles.

\subsection{Effets complexes de l'empreinte climatique entraînant une baisse des rendements rizicoles}

L'analyse de la situation pluviométrique à la station de Sédhiou révèle une très forte variabilité dans la zone d'étude (Figure 2). En effet, cette variabilité, accentuée en Moyenne Casamance et dans les régions Ouestafricaines a entraîné une remise en question des schémas traditionnels d'une agriculture se contentant essentiellement de la pluie (Manzelli et al., 2015). Ainsi, l'accroissement des températures et la variabilité annuelle des pluies représentent-elles une menace sérieuse pour le développement agricole, notamment de la riziculture et compromettent-elles les efforts déployés par l'État et ses partenaires pour faire face à l'insécurité alimentaire. La variabilité pluviométrique, associée à la hausse des températures, entraîne des dysfonctionnements sur les saisons agricoles, lesquels passent par des perturbations des cycles biologiques des cultures, occasionnant une détérioration de la production. Or, la riziculture pluviale de bas-fond en Moyenne Casamance est tributaire du régime pluviométrique ou de la saison pluvieuse qui s'étale sur 5 mois. Le constat est qu'il y a une dégradation des conditions pluviométriques et la mauvaise répartition des pluies dans le temps et dans l'espace avec un climat de type soudano-sahélien oscillant entre les isohyètes 1 200-1 500 mm de pluie par an. Entre 1984 et 2011, on est passé de $1072 \mathrm{~mm}$ enregistrés à $800 \mathrm{~mm}$ en moyenne avant d'atteindre plus de 1400 mm en 2012 (Figure 2). 


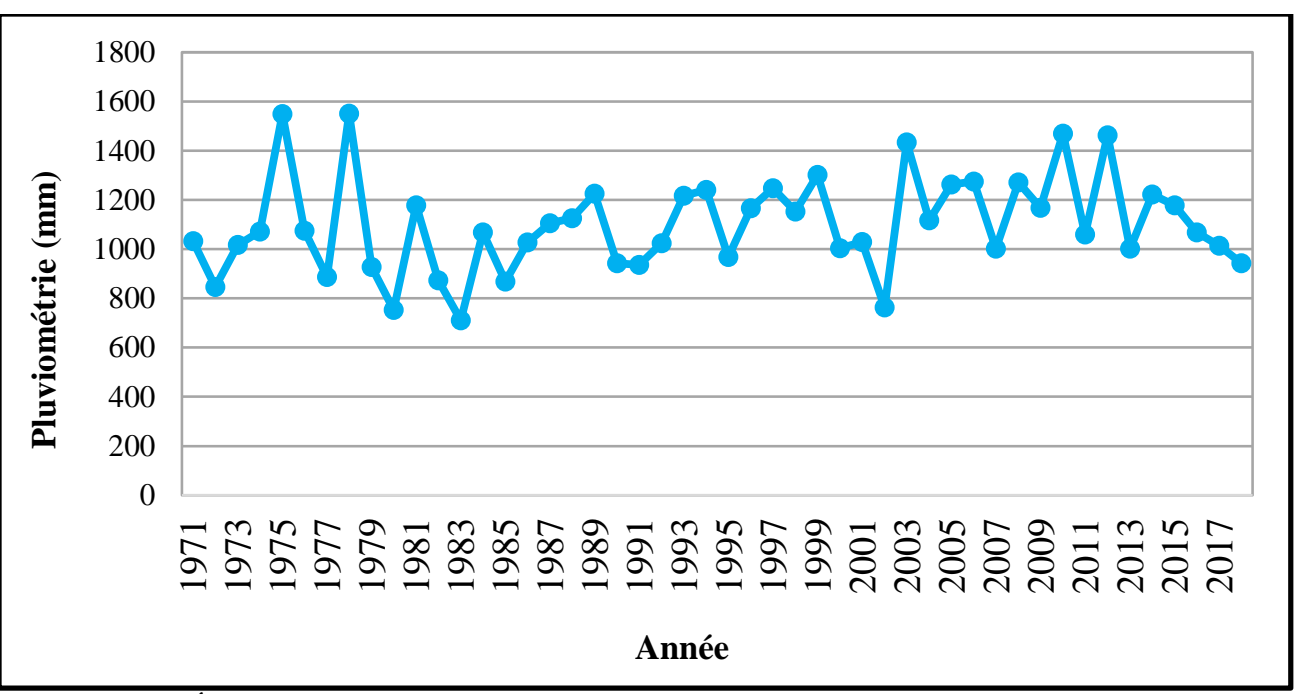

Figure 2 : Évolution annuelle de la pluviométrie à la station de Sédhiou de 1971 à 2017

Source : Résultats de l'enquête, 2021

La figure 2 montre la variabilité des précipitations dans la région de Sédhiou. Elles sont généralement comprises entre 800 et $1400 \mathrm{~mm}$ par an. Ces précipitations ont des impacts sur la production rizicole et amènent les rizicultrices à associer la production rizicole à d'autres cultures comme le mil et l'arachide. En effet, ces impacts se lisent à travers des pluies intenses et de courtes durées souvent enregistrées. Elles sont très favorables aux inondations, notamment dans certaines parties de la vallée, et peuvent paralyser la pratique de la riziculture. Il y a aussi l'état défectueux des ponts-barrages et des digues censées retenir l'eau lors des excédents pluviométriques. Les productrices mettent en place des techniques très élaborées pour la gestion de l'eau en rizières des zones de bas-fonds. Pour une gestion efficace des ouvrages, les vannes sont fermées en début de saison des pluies puis ouvertes une fois que les parcelles sont inondées pour évacuer le sel. Cette technique d'aménagement rizicole se traduit par la mise en place de séries de digues et de diguettes. Ce type d'aménagement facilite le lessivage des sels et acides, et permet un meilleur contrôle du niveau des plans d'eau (Sané, 2017). Finalement, ces digues traditionnelles permettent d'atténuer l'intrusion des eaux marines pour une meilleure gestion des parcelles rizicoles; cette situation contribue à la baisse des rendements. Cependant, les années de déficits pluviométriques (moins fortes au Sud) ont marqué les rendements par rapport aux besoins des populations. Cette riziculture subit les incidences des changements climatiques (variabilité des pluies, salinisation des terres, l'apparition d'herbes envahissantes, pauvreté du sol etc.) qui remet en question toutes les certitudes des paysans. De plus, cette variabilité pluviométrique laisse son incidence sur toutes les autres données climatiques. Les 
températures moyennes mensuelles les plus basses sont enregistrées entre décembre et janvier et varient entre 25 et $30^{\circ} \mathrm{C}$. Les plus élevées sont notées entre mars et septembre avec des variations de 30 à $40^{\circ} \mathrm{C}$ (Manzelli et al., 2015). Cette variabilité pluviométrique influence l'écoulement de l'eau dans les vallées rizicoles.

\subsection{Influence du régime hydrographique du fleuve Casamance sur la salinisation des rizières}

Le fonctionnement en estuaire inverse du fleuve Casamance influe sur la culture du riz. En effet, la proximité du fleuve entraîne l'avancée du sel. À cela, s'ajoute la baisse de la pluviométrie ces dernières décennies et celle de l'écoulement fluvial qui ont exacerbé le fonctionnement de l'estuaire de la Casamance : les concentrations de sel ont dépassé $100 \mathrm{~g} / \mathrm{l}$ dans les hauts estuaires (Thior et al., 2019). Selon les enquêtes réalisées, la problématique la plus récurrente évoquée par les rizicultrices est la salinisation (61\%). Aussi, l'ensablement (30\%) et l'acidification ( $9 \%$ ) représentent un problème central. L'acidification vient du déficit pluviométrique, du rabattement des aquifères et de l'oxydation des sulfures. C'est dans cette lancée que le Projet de Gestion des Eaux en zone Sud (PROGES) a construit une digue anti-sel en aval de la vallée (village de Binako chérif) pour lutter contre la salinisation des rizières. La dégradation des sols a des effets sur le rendement, et des rizières sont devenues non cultivables dans la zone. La principale conséquence est la baisse progressive de la productivité rizicole. Par exemple, les rendements moyens qui atteignaient $500 \mathrm{~kg}$ de paddy sur une parcelle de $500 \mathrm{~m}^{2}$ entre 1996-1999, soit des rendements très performants connaissent une baisse par rapport aux résultats obtenus entre 2000 et 2004 soit $300 \mathrm{~kg}$ pour $500 \mathrm{~m}^{2}$. En outre, sous l'effet du ruissellement des eaux pluviales, beaucoup de rizières ont été envahies par le sable. Dans la vallée, la dégradation des sols s'est accentuée avec l'apparition de ravins. Malgré la densité de la végétation, le phénomène n'épargne pas les rizières (Photo 1). 
Photo 1 : Dégradation et effet du ravinement d'une partie de la vallée de Badobar

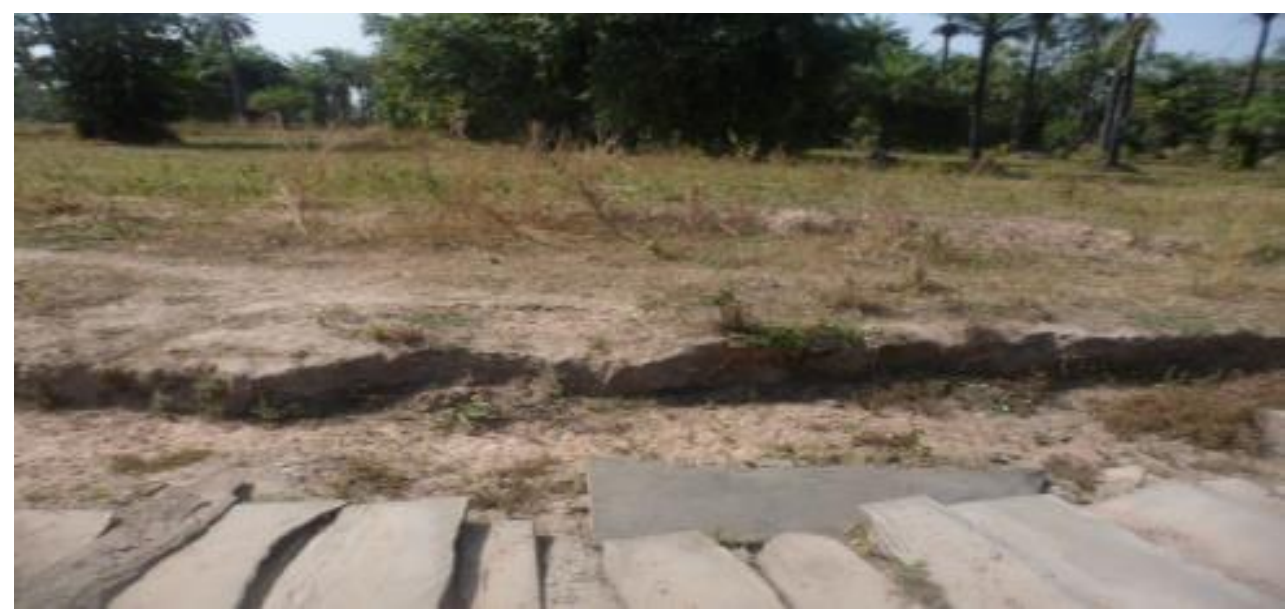

Source : Données de l'enquête, 2019

La photo 1 montre l'effet du ravinement causé par les eaux de ruissellement dans la vallée obligeant les rizicultrices à mettre en place des planches pour éviter ou diminuer l'érosion qui affecte les périmètres rizicoles. Malgré cet effort, 55\% des rizières de la vallée de Badobar sont envahies par l'ensablement. Ce phénomène constitue une forme de dégradation des sols qui s'accentue et freine l'élan de la production rizicole. Comme l'indique une rizicultrice rencontrée en 2018 dans la vallée :

"L'ensablement a fortement réduit les surfaces que je cultive. À cause du dysfonctionnement des digues ou diguettes, l'ensablement agresse fortement mes parcelles. Ce phénomène est causé par le ruissellement des pluies créant parfois des ravins. Ces ravins deviennent des zones de dépôt de sable, ce qui rend impossible la pratique de la riziculture dans la vallée. En 2018, j'étais obligée d'abandonner 2 parcelles qui étaient devenues inexploitables. Cette situation est à l'origine de la diminution de mes rendements de riz. Par exemple, en 2018j'ai obtenu des rendements faibles, soit $200 \mathrm{~kg}$ de riz paddy sur une superficie de $500 \mathrm{~m}^{2} »$.

En définitive, les causes liées aux changements climatiques sont entre autres: la variabilité pluviométrique, l'ensablement, l'acidification des rizières et la remontée de la langue saline liés au réseau hydrographique du fleuve Casamance etc. (Tableau I). 
Tableau I : Les causes environnementales de la dégradation des rizières de la vallée de Badobar

\begin{tabular}{|c|c|c|}
\hline Phénomènes & $\begin{array}{c}\text { Causes liées aux } \\
\text { changements climatiques }\end{array}$ & Conséquences \\
\hline $\begin{array}{l}\text { Ruissellement des eaux de } \\
\text { pluie des plateaux vers la } \\
\text { vallée }\end{array}$ & - $\quad$ Forte pluviométrie & $\begin{array}{c}\text { - } \quad \text { Ravinement } \\
\text { - Ensablement des rizières } \\
\text { - Inondation dans certaines } \\
\text { parties des rizières } \\
\text { - } \quad \text { Abandon des rizières } \\
\end{array}$ \\
\hline $\begin{array}{l}\text { Ensablement des rizières } \\
\text { de la vallée }\end{array}$ & $\begin{array}{c}\text { - Phénomènes érosifs } \\
\text { Inefficacité des ouvrages hydro } \\
\text { agricoles }\end{array}$ & $\begin{array}{c}\text { - Transport de particules de } \\
\text { sables fins des plateaux vers } \\
\text { la vallée } \\
\text { - } \quad \text { Abandon de parcelles } \\
\text { - } \quad \text { Réduction des surfaces } \\
\text { cultivables } \\
\end{array}$ \\
\hline Salinisation des rizières & $\begin{array}{c}\text { Remontée de la langue salée } \\
\text { liée au réseau hydrographique } \\
\text { du fleuve Casamance }\end{array}$ & $\begin{array}{l}\text { - } \quad \text { Abandon des rizières } \\
\text { - } \quad \text { Eau saumâtre } \\
\text { - Baisse des rendements } \\
\text { - Baisse de la qualité de l'eau }\end{array}$ \\
\hline Acidification & Diminution de la pluviométrie & - $\quad$ Abandon des rizières \\
\hline
\end{tabular}

Source : Résultats de l'enquête, 2021

Pour autant, des conditions socio-économiques des agricultrices constituent des facteurs limitant le développement de la riziculture. Dès lors, il est intéressant de s'interroger d'abord sur le rôle central que jouent les femmes dans la pratique de la riziculture pluviale.

\subsection{Des femmes, forces motrices de la riziculture pluviale et garantes de l'autoconsommation familiale}

Contrairement à la Basse Casamance où les hommes interviennent dans les exploitations rizicoles (Diédhiou et al., 2019), en Moyenne Casamance la culture du riz est l'apanage des femmes. En pays mandingue comme dans le pays balante, ce sont les femmes qui dominent dans la production du riz. Les femmes $(90 \%)$ sont les plus impliquées dans la phase de production de riz. Dans la vallée, $70 \%$ des rizicultrices ont entre 25-50 ans et $30 \%$ plus de 50 ans. Majoritairement avec un niveau de scolarité plutôt faible : $62 \%$ n'ont pas fréquenté l'école, $26 \%$ le primaire et $12 \%$ le niveau moyen ou secondaire dont $8 \%$ à l'école arabe. Les ethnies les plus représentatives dans l'activité rizicole sont: les Mandingues (65\%), les Balantes (15\%), les Manjacks (10\%), les Mancagnes (5\%) et les Peuls (5\%). Plus spécifiquement, les femmes assurent toutes les tâches allant de la préparation de la rizière à la récolte en passant par la transformation manuelle des brisures (Photo 2). Les rizicultrices utilisent des matériels rudimentaires tels que le coupe-coupe (15\% des cas), le couteau ( $20 \%$ des cas), le loto (un 
instrument traditionnel fabriqué par des artisans locaux pour le repiquage du riz) [30\% des cas] et le baro (un instrument traditionnel constitué par une lame de daba avec lequel les productrices manient la terre) [35\% des cas], ce qui explique qu'elle nécessite la présence de la main-d'œuvre familiale, de beaucoup de temps et d'efforts physiques (Photo 2).

Photo 2 : Des femmes assurant le travail pénible de pilage du riz paddy pour le décortiquer

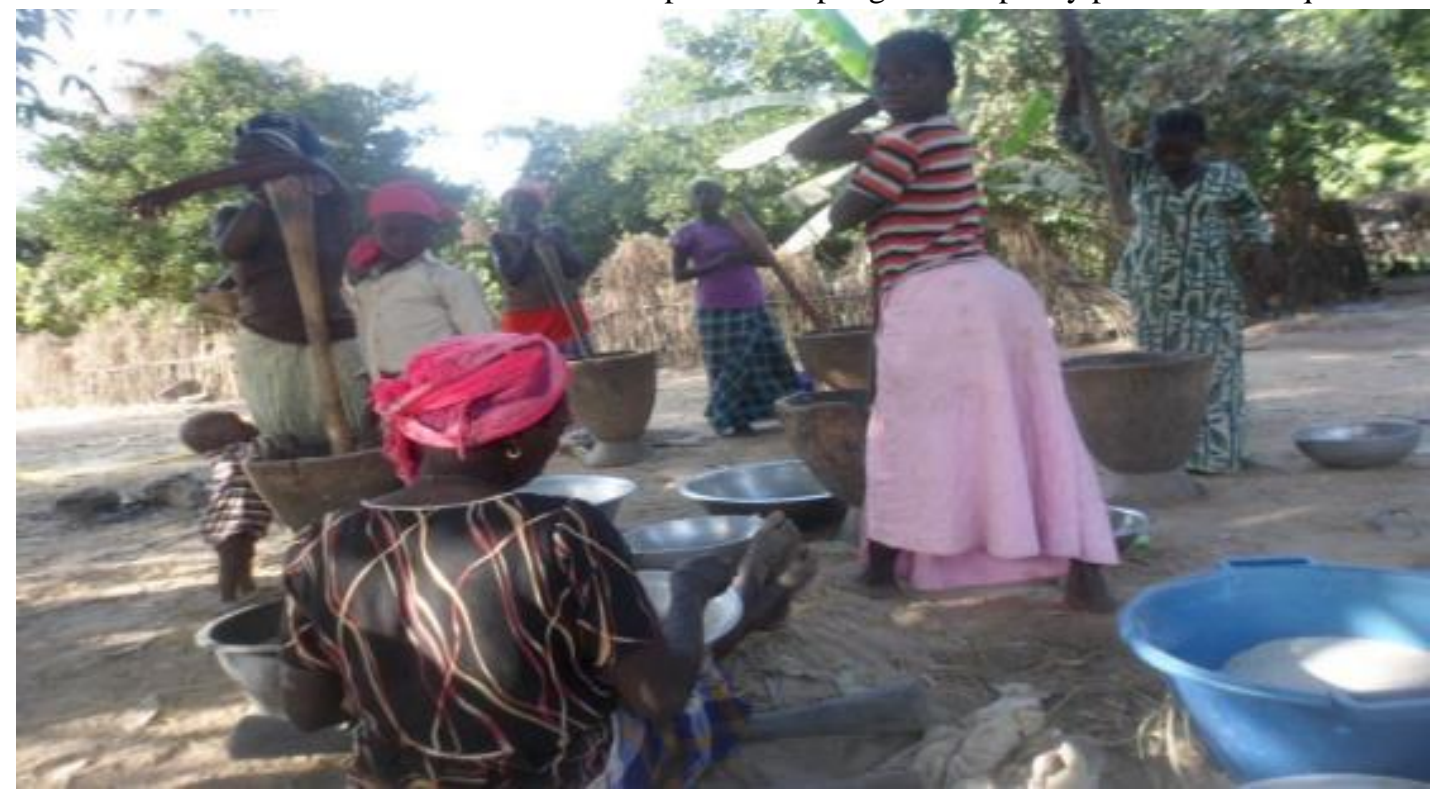

Source : Données de l'enquête, 2019

La photo 2 montre un groupe de femmes composé d'une rizicultrice et ses enfants décortiquant le riz paddy pour sa transformation en riz blanc. Cet exercice de décorticage du paddy se fait à l'aide de matériels rudimentaires, notamment des mortiers et des pilons qu'elles manient debout avant de trier les mauvaises graines. Ce riz est exclusivement destiné à l'autoconsommation familiale. Par contre, les femmes rencontrent des difficultés pour manier des outils lourds. Une productrice rencontrée en 2018 indique que : « ce travail de décorticage est pénible. J'ai des courbatures au dos et des douleurs musculaires ». Par ailleurs, elles assurent le semis, le repiquage et effectuent les opérations de désherbage, d'épandage, de battage, de vannage et de transport des récoltes. La coupure des épis de riz, la transformation des produits et le pilage les concernent exclusivement. En définitive, cette féminisation de la culture du riz, contrairement en Basse Casamance où les hommes s'y impliquent, se répercute sur les rendements.

Les productrices entretiennent des pépinières de riz et construisent des diguettes. Elles peuvent parfois compter sur la main-d'œuvre familiale. La participation des femmes aux travaux agricoles fait partie de leurs obligations familiales. La disparité homme-femme dans la pratique de la riziculture dans 
ces villages peut expliquer la faiblesse des rendements. En effet, les femmes ne parviennent pas à réaliser certains aménagements hydroagricoles qui demandent des efforts physiques. De ce fait, $70 \%$ des rizicultrices interrogées déplorent l'absence des hommes dans toutes les phases culturales. Parallèlement, les hommes assurent des cultures pluviales de céréales (arachide, mil, sorgo, maïs, fonio) dans des parcelles de taille comprise entre 300 et $500 \mathrm{~m}^{2}$, souvent dans les zones de plateau. Les hommes apportent aussi leur soutien financier aux productrices pour le recrutement de la main-d'œuvre d'une part, et participent aux travaux manuels tels que le labour complet des parcelles rizicoles puis la récolte du riz d'autre part. Cependant, il est indispensable de mesurer la contribution des femmes dans la mise en valeur de la terre à travers ces différentes activités, pour comprendre les facteurs socioéconomiques qui freinent le développement de la riziculture pluviale, les enjeux véritables des relations tissées à l'intérieur de la cellule économique qui est l'exploitation foncière, familiale, productrice de riz (Diédhiou et al., 2018 ; Sow, 1992).

\section{Facteurs socioéconomiques bloquant le développement de la riziculture dans la vallée de Badobar}

En Moyenne Casamance, l'accès à la terre, le manque de financement, la faiblesse de renouvellement de la main-d'œuvre et des ouvrages hydroagricoles constituent des limites au développement de l'activité rizicole.

\subsection{Un mode d'accès à la terre désavantageux aux rizicultrices}

Le foncier est une question épineuse au Sénégal, surtout dans un contexte de raréfaction de la ressource (Vennetier, 1989). Dans la vallée de Badobar, le régime foncier est de type traditionnel. Plusieurs solutions coexistent pour accéder à la terre ${ }^{1}$ : héritage $(95 \%)$, prêt (27\%), location (4\%) et achat $(1 \%)$.

Les résultats montrent que l'héritage constitue la modalité la plus fréquente puisqu'il est le recours retenu de $95 \%$ de rizicultrices. Cette modalité est basée sur une garantie de propriété écrite et légalisée. En effet, les rizières sont des propriétés familiales et c'est le chef de famille qui s'occupe de leur gestion. Ce dernier peut procéder à la location, au prêt ou à la vente des terres. Pour l'exploitation, seuls les garçons bénéficient des terres après mariage alors que les filles n'ont pas accès à la propriété des terres rizicoles. Dans de rares cas, les femmes peuvent bénéficier de terre quand leur mari décède. Dans un second registre, $27 \%$ de rizicultrices ont recours au prêt. Dans ce cas, il n'existe aucun contrat écrit, mais plutôt un accord oral entre le propriétaire de

1 Les fréquences de citation sont supérieures à 100, cette situation s'explique par le fait que des femmes accèdent de différentes manières à la terre : soit par héritage soit par prêt. 
la parcelle et l'intéressée. Souvent, cette modalité se traduit par l'interconnaissance issue de famille ou du voisinage. Enfin, la modalité liée à la location est activée par une part faible des rizicultrices (4\%). Le montant de la location varie entre 10000 et 15000 F.CFA et se fait sur des superficies comprises entre 200 et $500 \mathrm{~m}^{2}$. Finalement, les femmes sont écartées de l'héritage, ce fait explique qu'elles ont une réelle difficulté à accéder au foncier agricole. Le paradoxe, dans la société Mandingue, les hommes héritent de la terre, mais la riziculture est pratiquée à $97 \%$ par les femmes. Par contre, les filles sont appelées à se marier hors de la maison familiale, donc elles héritent généralement des parcelles de la famille d'accueil. Cette féminisation de la riziculture influe sur le statut économique des productrices marqué par une crise dans le monde rural, une baisse de la production et des profits.

Pour autant, la riziculture pluviale est une activité exclusivement destinée à l'autoconsommation, par exemple 98\% des interrogées indiquent conserver toute leur production à l'alimentation familiale. La production comble $45 \%$ des besoins alimentaires et couvre les besoins de consommation familiale durant 4 ou 5 mois de l'année. Ce résultat atténue en partie les dépenses en riz importé. Cependant, $5 \%$ des femmes pratiquent en parallèle le maraîchage. Les produits (aubergine amère, gombo, oseille, tomate, navet, laitue etc.) issus de cette activité sont commercialisés au niveau des marchés. Les revenus moyens mensuels varient de 50000 à 90000 F.CFA et constituent un complément pour assurer les autres dépenses alimentaires et courantes familiales (scolarité des enfants, achat de riz parfumé etc.). Par ailleurs, la cueillette et l'exploitation de la noix de cajou constituent une activité connexe qui permet aux femmes d'avoir des revenus supplémentaires. Malgré ce dynamisme, les femmes rencontrent des difficultés pour recourir à un financement.

\subsection{Manque de financement, un facteur contraignant à la production rizicole}

Les contraintes économiques rencontrées par les rizicultrices freinent des investissements conséquents pour une bonne production de riz. Les résultats issus des enquêtes de terrain révèlent que $95 \%$ des femmes n'ont pas de sources de financement. Ces dernières comptent sur les moyens financiers mis en place par l'État à travers les projets et programmes de distribution de semences et de matériels agricoles. D'ailleurs, $45 \%$ des femmes interrogées affirment directement consommer la moitié des semences destinées à la production. Il peut en résulter la difficulté d'accès aux intrants, aux équipements ou au crédit. De plus, $55 \%$ des femmes n'arrivent pas à se procurer de fertilisants ou de produits phytosanitaires afin de lutter contre les ravageurs et maladies affectant les plantes. Ce manque de moyens financiers 
est à l'origine de l'utilisation d'outils rudimentaires dans toutes les phases de la production (Tableau II).

Tableau II : Causes anthropiques de la dégradation des rizières dans la vallée de Badobar

\begin{tabular}{|c|c|c|}
\hline Phénomènes & Causes anthropiques & Conséquences \\
\hline $\begin{array}{l}\text { Absence de revenus des } \\
\text { agricultrices }\end{array}$ & $\begin{array}{c}\text { - Pauvreté économique des } \\
\text { agricultrices } \\
\text { - } \quad \text { Absence de financement } \\
\end{array}$ & $\begin{array}{ll}\text { - } & \text { Insécurité alimentaire } \\
\text { - } & \text { Exode rural des jeunes }\end{array}$ \\
\hline $\begin{array}{l}\text { Renouvellement de la main- } \\
\text { d'œuvre }\end{array}$ & Vieillissement des agricultrices & $\begin{array}{l}\text { - } \quad \text { Abandon des rizières } \\
\text { Diminution de la production }\end{array}$ \\
\hline $\begin{array}{l}\text { Mauvaise gestion des ouvrages } \\
\text { hydroagricoles }\end{array}$ & $\begin{array}{cc}\text { - } & \text { Faible soutien de l'État } \\
\text { - } & \text { Absence d'entretien des } \\
\text { infrastructures }\end{array}$ & $\begin{array}{l}\text { Réduction de la productivité } \\
\text { rizicole } \\
\text { - } \quad \text { Insécurité alimentaire } \\
\text { - } \quad \text { Ensablement des rizières }\end{array}$ \\
\hline $\begin{array}{l}\text { Manque d'encadrement des } \\
\text { rizicultrices }\end{array}$ & $\begin{array}{c}\text { - } \begin{array}{c}\text { Insuffisance de personnel } \\
\text { technique qualifié }\end{array} \\
\text { - } \quad \text { Faiblesse des moyens } \\
\text { logistiques } \\
\text { Absence de systèmes de suivi } \\
\text { des activités de formation }\end{array}$ & $\begin{array}{c}\text { - } \quad \text { Adoption de pratiques } \\
\text { autochtones } \\
\text { - } \quad \text { Utilisation de moyens et } \\
\text { matériels rudimentaires } \\
\text { - Périodisation non calculée }\end{array}$ \\
\hline Difficulté d'accès aux intrants & $\begin{array}{c}\text { Faiblesse des revenus des } \\
\text { agricultrices } \\
\text { - } \quad \text { Absence de financement }\end{array}$ & Diminution des rendements \\
\hline Divagation des animaux & $\begin{array}{c}\text { Absence de clôture des parcelles } \\
\text { Absence de pâtures pour les } \\
\text { animaux }\end{array}$ & Baisse de la productivité \\
\hline
\end{tabular}

Source : Résultats de l'enquête, 2021

De manière générale, la majorité des femmes demande un appui de l'État et des partenaires au développement afin d'améliorer les conditions de travail dans les rizières. Comme l'indique une rizicultrice rencontrée en 2018 dans la vallée :

"Je ne dispose pas assez d'argent me permettant d'acheter des intrants agricoles. Cette situation explique que j'utilise des matériels traditionnels comme le baro, le couteau, la daba etc. pour mettre en valeur ma parcelle. Je fractionne parfois mes dépenses pour l'achat de produits phytosanitaires. Ces difficultés financières associées aux conditions pédologiques et climatiques font que j'ai des problèmes pour obtenir un bon rendement. La plupart $d u$ temps, je compte sur les semences distribuées par l'État pour pouvoir pratiquer la riziculture». 


\subsection{Faiblesse de renouvellement de la main-d'ouvre et des ouvrages hydroagricoles vétustes, facteurs limitant la productivité rizicole}

Le faible renouvellement de la main-d'œuvre pose une problématique de production. L'âge moyen des rizicultrices est compris entre 45 et 60 ans, soit $70 \%$ des cas dans la vallée de Badobar. Ce vieillissement des rizicultrices pose un problème de renouvellement générationnel. En effet, les plus jeunes composés d'étudiants et de fonctionnaires ne participent presque pas à la riziculture. Cette situation est à l'origine du problème de relève de la maind'œuvre. Les enquêtes de terrain prouvent que la tranche d'âge comprise entre 25 et 35 ans ne représente que $15 \%$ de la main-d'œuvre. Néanmoins, les $25 \%$ des rizicultrices ont recours à la main-d'œuvre salariée. Le paiement de cette main-d'œuvre est assuré par l'argent issu de la vente de produits maraîchers (laitue, chou, gombo, oseille etc.). De plus, les entretiens révèlent qu'après la phase de récolte, certaines productrices pratiquent le système de troc c'est-àdire rémunérer la main-d'œuvre moyennant du riz. Dans de rares cas, la rémunération peut émaner de leurs maris et parfois même d'autre membre de la famille (oncle, neveux, tante etc.). Par ailleurs, certaines femmes s'organisent en groupements d'intérêt économique (GIE) communément appelé « dahira » en Mandingue et « société » chez les Peuls pour s'entraider. En effet, la location des paires de bœufs pour le labour complet d'une parcelle rizicole de $500 \mathrm{~m}^{2}$ varie entre 2000 et 4000 F.CFA alors que le décorticage du riz est évalué à 100 F.CFA le kilogramme. En outre, le système de troc est le moyen de paiement le plus utilisé pour rémunérer la main-d'œuvre qui transporte la production de riz des champs à la maison. En effet, pour un chargement de charrette de $300 \mathrm{~kg}$ de riz transporté, un prestataire reçoit $5 \mathrm{~kg}$ de riz.

Néanmoins, les rizicultrices de la vallée de Badobar ont bénéficié de projets agricoles (État, ONG, partenaires au développement etc.) par la mise en place d'ouvrages hydroagricoles qui sont depuis les années 1990 dans une vétusté avancée. Pour donner suite à la relance de la riziculture pluviale, le projet de gestion des eaux du Sud (PROGES) et le Projet Pôle de Développement de la Casamance (PPDC) ont réalisé des aménagements hydroagricoles avec la construction de six digues ${ }^{2}$ dans la zone.

Toutefois, depuis une décennie, l'absence de gestion par les autorités locales et des paysans a entrainé la détérioration des infrastructures hydroagricoles, notamment les digues. Cette situation est à l'origine de la diminution des rendements rizicoles. Par exemple, des vannes ont été emportées par les forts débits des eaux de ruissellement (Photo 3). Un second registre montre la mise en place d'une digue anti-sel permettant d'empêcher

${ }^{2}$ L'État à travers les services déconcentrés (DRDR, SDDR et service de l'agriculture) et les partenaires au développement (PAM) construit les infrastructures hydroagricoles. 
la remontée de l'eau salée du fleuve Casamance vers les rizières. En amont, la digue ralentit le dessèchement des sols de bas-fonds, tout en contribuant à atténuer le phénomène d'acidification. En aval cette digue permettait l'inondation des rizières durant la saison pluvieuse. Malgré l'établissement de ces infrastructures hydroagricoles, le 1/3 des parcelles rizicoles est conquis par le sel et n'est plus propice à la riziculture.

Photo 3 : Digue de rétention d'eau de Beylan dans un état vétuste

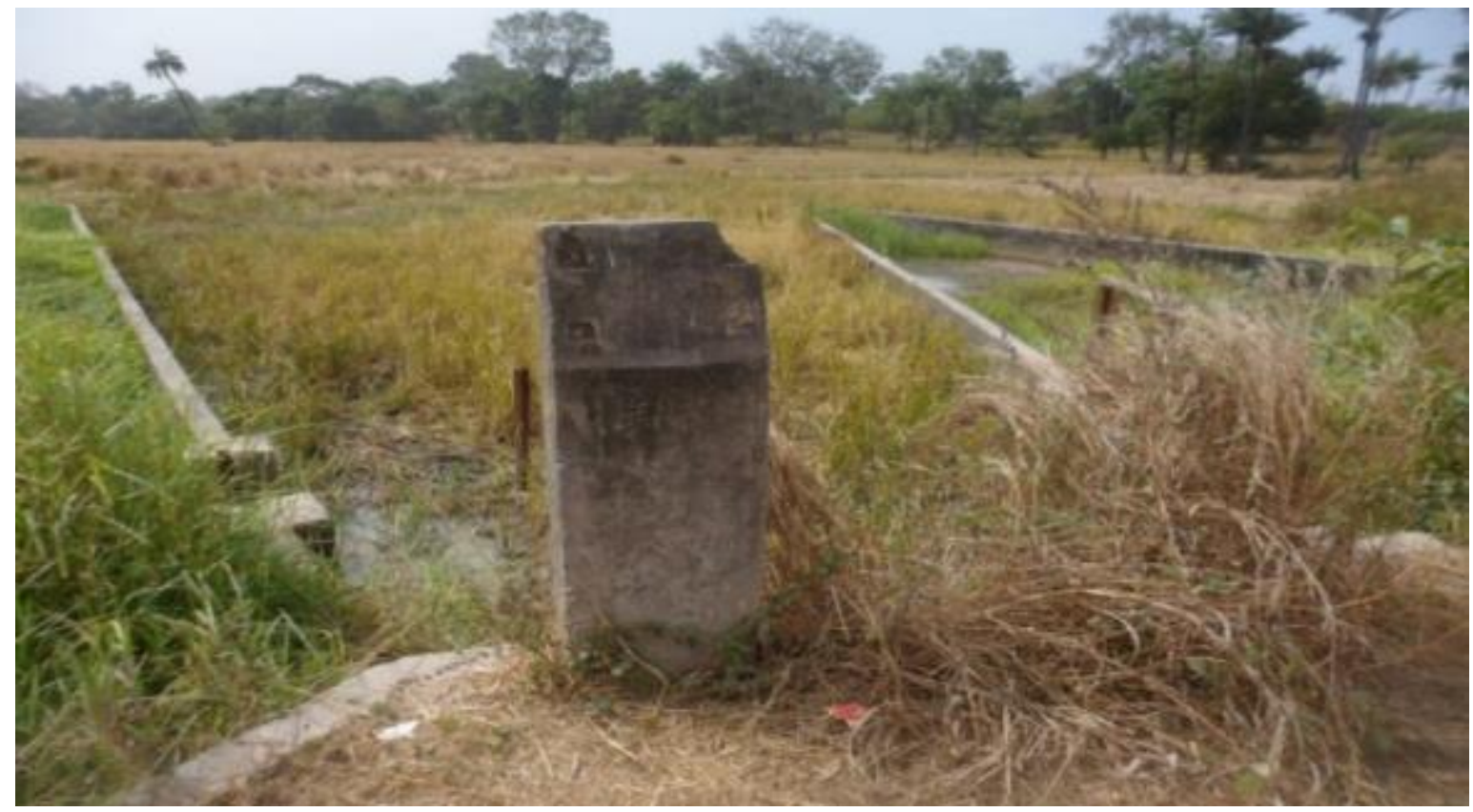

Source : Données de l'enquête, 2019

Au total, l'ensablement et l'érosion hydrique sont de plus en plus inquiétants dans cette partie de la vallée (Photo 3). Plus spécifiquement, 55\% des rizicultrices abandonnent leur parcelle à cause de l'ensablement contre $45 \%$ du fait de l'érosion hydrique. Il est remarqué un dessèchement des sols, mais aussi l'acidification des rizières (photos $4 \mathrm{a}$ et $4 \mathrm{~b}$ ). Cette dégradation de l'environnement est à l'origine de la faiblesse des rendements. Comme l'indique une rizicultrice rencontrée en 2018 dans la vallée :

"Dans les années 1990, le fonctionnement correct des digues de rétention d'eau favorisait l'inondation de ma parcelle. Je réalisais des rendements estimés entre 600 et $750 \mathrm{~kg}$ de riz paddy dans un périmètre de 500 $m^{2}$. Malheureusement, depuis les années 2000 avec l'état défectueux des digues, l'ensablement est devenu une problématique majeure. Les vannes ne sont plus fermées et certaines ont été volées par des habitants 
du village. Pour moi, ces raisons ont entraîné la baisse de mes rendements estimés entre 400 et $500 \mathrm{~kg}$ de riz paddy dans une même parcelle de $500 \mathrm{~m}^{2}$ ».

Photo 4 : L'état des infrastructures hydroagricoles dans la vallée de Badobar

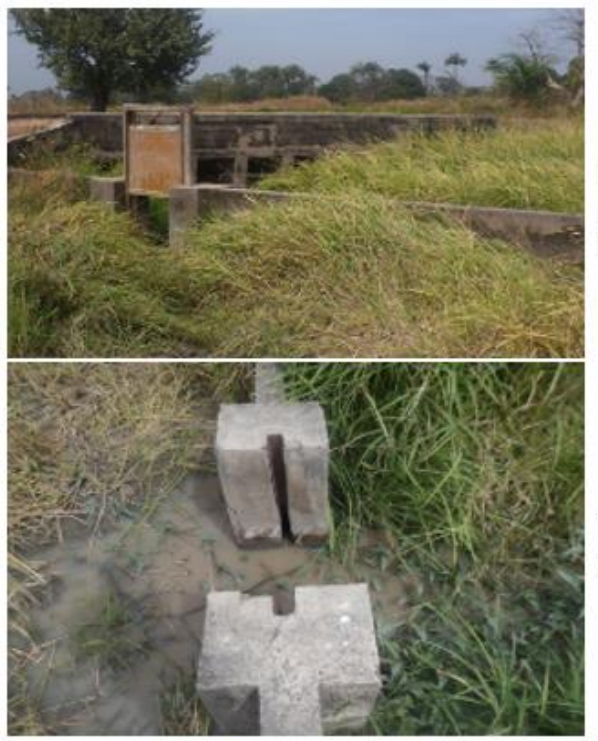

Photo (4a): Ouverture de la vanne à un moment pas propice entrainant un problème de gestion et de rétention d'eau.

Source : Données de l'enquête, 2019

La photo (4a) montre une vanne ouverte, ce qui révèle que les ouvrages ne sont plus entretenus alors que la gestion de l'eau est primordiale pour une riziculture pluviale. La photo (4b) montre que l'absence de vanne de protection est à l'origine de l'assèchement des parcelles entraînant le manque de productivité rizicole. En définitive, le manque d'entretien et de suivi des infrastructures construites entraîne leur dégradation progressive.

Pour pallier cette problématique, les populations, notamment les rizicultrices ont développé plusieurs stratégies d'adaptations.

\section{Des stratégies communautaires ingénieuses déployées pour atténuer localement les effets des changements climatiques}

Dans un souci d'atteindre l'autosuffisance en riz dans la zone, de nouvelles stratégies ont été adoptées. Pour ce faire, les productrices adoptent de nouvelles variétés de riz à cycle court pour répondre à la variabilité pluviométrique afin d'améliorer les rendements. En outre, d'autres stratégies et techniques sont préconisées pour l'amélioration de la production ainsi qu'à la construction de digues pour protéger les vallées. 


\subsection{Introduction de nouvelles variétés de riz à cycle court, une exigence pour l'amélioration des rendements}

Les femmes ont développé diverses stratégies pour atténuer les effets des changements climatiques et anthropiques. L'amélioration de la production passe par une introduction de variétés rizicoles et de fertilisants. Ainsi, 66\% des rizicultrices interrogées ont recours à une nouvelle variété de riz. Ces variétés ont été introduites dans la vallée en 1994 par le PROGES. De ce fait, $34 \%$ des rizicultrices ont recours à l'association de variétés à cycle court. Les variétés adoptées sont majoritairement d'origine locale (région de Sédhiou). Parfois, certaines variétés peuvent provenir de pays frontaliers comme la Gambie ou des régions de l'intérieur. Dans $90 \%$ des cas, l'espèce adoptée porte le prénom de la personne qui est à l'origine de son adoption ou celui de la localité d'origine (Mendy, 2018 ; Diallo, 2014). De manière générale, ces stratégies (association de variétés à cycle court, usage de fertilisations organiques ou chimiques) s'avèrent efficaces pour l'amélioration de la production. Comme l'indique une productrice rencontrée en 2019 dans le village de Beylan :

"Depuis que j'ai commencé à utiliser les nouvelles variétés de cycle court, ma production a augmenté. Durant la campagne 2017-2018, j'ai réussi à obtenir des rendements estimés entre 700 et $800 \mathrm{~kg}$ de riz paddy dans une parcelle de $500 \mathrm{~m}^{2} »$.

Parallèlement, les productrices ont recours à des fertilisants chimiques et organiques. Les techniques couramment citées sont l'usage de fumures d'animaux (moutons, bœufs, volailles), de l'urée et de cendre issus de brulis de feuille de manguiers. Ce système occupe une place centrale dans le processus d'amendement du sol. L'usage de fumier d'élevage est pratiqué par $45 \%$ des répondants alors que $35 \%$ de celles-ci déclarent recourir à l'urée, et $20 \%$ des productrices ont recours à l'usage de cendre issue de feuilles de manguiers. Ces techniques traditionnelles sont efficaces et permettent de garder la fertilité du sol et de surcroît, de produire en quantité, du riz destiné à l'autoconsommation.

\subsection{Organisations paysannes, une alternative pour une meilleure prise en charge des préoccupations des rizicultrices}

L'ensemble des acteurs concernés (rizicultrices, État, ONG etc.) déploie des stratégies individuelles et/ou traditionnelles et des stratégies collectives. C'est ainsi que le gouvernement du Sénégal a mis en place des projets et programmes pour relancer la riziculture pluviale dans la région de Sédhiou. Il s'agit entre autres du Programme d'Appui au Programme National d'Investissement de l'Agriculture du Sénégal (PAPSEN), du Projet de Gestion 
des Eaux en Zone Sud (PROGES), du Programme Pôle de Développement de la Casamance (PPDC) etc. Ces derniers sont financés par l'État du Sénégal en collaboration avec de partenaires et organismes internationaux et sousrégionaux (USAID, FAO, AfricaRice etc.). Ainsi, plusieurs actions sont entreprises pour assurer un renforcement de la productivité rizicole, une meilleure gestion des eaux pluviales, une bonne formation des productrices et surtout pour supporter la récupération des sols salinisés et la riziculture dans la vallée de Badobar. D'ailleurs, ces organismes ont mis en place des projets et programmes agricoles et de renforcement de capacité des productrices. Par exemple, le PROGES a formé 47 productrices, dont 21 femmes dans 18 villages. Ces projets et programmes appuient les paysans et paysannes en les formant en alphabétisation, à la technique de compostage, à la fabrication de grillages et autres. La mise en place du Comité de gestion des ressources de la vallée de Badobar (COGERBA) regroupant un comité inter-villageois est un élan de développement dans la zone. Ce sont les productrices qui sont à l'initiative de la création de ce comité de gestion. L'objectif était de réunir tous les villages riverains de la vallée pour œuvrer au développement socioéconomique de leur terroir. Les résultats issus des présents travaux de terrain révèlent qu'en 2018 ce comité disposait d'une somme de 167000 F.CFA qui a permis de passer une commande de $885 \mathrm{~kg}$ d'engrais pour les productrices afin d'amender les parcelles rizicoles de $500 \mathrm{~m}^{2}$.

De plus, le regroupement en association villageoise ou Organisation Paysanne (OP) permet aux productrices de s'entraider. En effet, 55\% des rizicultrices sont organisés en OP, ainsi il y a une personne morale autour de laquelle tout se passe. Ces OP participent à tous les travaux rizicoles (semis, repiquage, récolte, battage). Elles se partagent le périmètre pour éviter une discrimination et chaque femme est obligée de se conférer aux règles établies par le groupe. Les travaux des femmes sont rémunérés soit en argent soit en riz pendant l'hivernage ou après les récoltes.

\section{Discussion}

La relation d'influence entre climat et riziculture constitue un intérêt scientifique visant à analyser les contraintes de production de la riziculture pluviale de bas-fonds et les stratégies d'adaptation des productrices dans la région de Sédhiou. Ainsi, comprendre les liens entre ces deux facteurs devient prégnant pour assurer le développement économique durable à l'échelle locale, voire nationale. Les recherches ont essentiellement prouvé que les productrices sont confrontées à des difficultés de production et mettent en place des stratégies d'adaptation durables (Thior et al., 2019 ; Mballo et al., 2019 ; Diédhiou et al., 2018 ; Mendy \& Sy, 2015 ; Manzelli et al., 2015).

Les résultats révèlent que dans ce terroir, la riziculture de bas-fond est traditionnellement plus répandue que la riziculture de plateau. De fait, avec 
une population à dominance mandingue (plus de 50\%), les villages sont effectivement répartis à leurs lisières, au-dessus des grandes vallées humides ou inondables. Les villages ont une position qui pourrait être celle de véritables villages de riziculteurs. La riziculture affirme son caractère «mandingue » dans l'ensemble de la paysannerie et occupe les bas-fonds et les petites vallées au prix d'aménagements mineurs tandis que les hommes se consacrent aux cultures de plateau, notamment le mil. C'est le même système de production qu'on retrouve chez les Balantes et Peuls, apanage des femmes avec des techniques et moyens parfois limités et identiques. Dans le terroir mandingue, on a un schéma qui forme relativement un triptyque «rizières, champs permanents et habitat de type paillote déplaçable ». On distingue deux modes de riziculture pluviale : la riziculture de bas-fond et la riziculture de plateau. La pratique de la riziculture dans la vallée repose sur des techniques autochtones de labour et de dessalement des sols, de repiquage et d'aménagement des casiers. Fondée naguère sur des variétés africaines, la riziculture a aujourd'hui adopté de nouvelles variétés plus productives. Mais le domaine des rizières reste confondu avec les vallées et les dépressions, où les femmes assument les rudes tâches du repiquage, du désherbage, de la surveillance, de l'inondation et de la récolte du paddy (Manzelli et al., 2015). En ce qui concerne les stratégies d'adaptions, les résultats du présent travail corroborent les travaux de Mendy $(2015,2018)$ qui ont montré que devant l'insuffisance des moyens matériels et financiers, les populations ont développé plusieurs stratégies - à côté de celles déployées par quelques organismes étatiques ou non - afin de minimiser le poids de la crise que traverse la riziculture locale. Le report de la riziculture vers le plateau et la construction de digues de protection sont des tentatives de réponses à la salinisation; alors que l'adoption de variétés à cycle court et moyen répond à une exigence liée au déficit pluviométrique. La majorité des digues sont munies d'ouvrages évacuateurs d'eau qui permettent des ouvertures et fermetures en fonction des besoins en eau (Mendy \& Sy, 2015). Plus spécifiquement, ces ouvrages permettent dans une certaine mesure, le lessivage des parcelles rizicoles surtout en amont de certaines rizières, mais aussi l'optimisation des besoins en eau des parcelles qui en sont déficitaires. C'est le cas des digues de Beylan, de Karoumbou, d'Adiouti et de Bissassou. Par ailleurs, les contraintes de production en riz pluvial ne sont pas spécifiques à la région de Sédhiou au Sénégal, elles s'observent ailleurs notamment en Afrique de l'Ouest. À titre illustratif, on peut citer les terroirs béninois et burkinabé (Kotchi et al., 2018 ; Atidegla et al., 2017 ; Traoré et al., 2015). Plus spécifiquement, Atidegla et al., (op. cit.) prouvent que le Bénin n'est pas épargné par la variabilité climatique qui engendre des contraintes de production en riz pluvial. Pour cet auteur, les conséquences de ces perturbations climatiques sur l'écosystème se traduisent par l'érosion des 
terres, l'inondation, le tarissement précoce des mares, les pertes de production, la baisse des rendements rizicoles etc. Pour limiter les effets des contraintes de production et améliorer leur bien-être, les productrices des vallées rizicoles ont développé des stratégies d'adaptation variables selon leurs catégories. Le présent résultat est en accord avec le constat de Traoré et al., (op. cit.) qui notaient qu'au Burkina Faso la riziculture pluviale offre un fort potentiel, mais est mal maîtrisée avec de faibles rendements. En effet, les rendements moyens sont d'environ $1075 \mathrm{~kg} / \mathrm{ha}$. La faible maîtrise de l'itinéraire technique de production nécessite une formation des producteurs pour accroître les rendements et booster la production du riz pluvial.

\section{Conclusion}

Le but de cet article est de comprendre les dynamiques actuelles et les enjeux de la riziculture pluviale dans la région de Sédhiou, en termes de contraintes de production et de stratégie d'adaptation. Dans un contexte marqué par les phénomènes des changements climatiques en Moyenne Casamance, la région de Sédhiou est caractérisée par une pluviométrie très variable. Cependant, la dégradation des rizières est liée à des facteurs physiques (salinisation, ensablement, érosion hydrique etc.) et de facteurs anthropiques (non-renouvellement de la main-d'œuvre, manque d'encadrement, absence des hommes dans la pratique de la riziculture, exode rural etc.). Au demeurant, la riziculture est de type traditionnel avec l'utilisation d'outils aratoires rudimentaires. Cette authenticité locale ou traditionnelle dans les pratiques culturales est une des difficultés qui influe sur le résultat en termes de productivité du riz. La combinaison de ces facteurs est à l'origine de la faiblesse des rendements sur les périmètres rizicoles de la vallée de Badobar. Les impacts sont aussi visibles à travers la diminution des surfaces rizicultivables et l'abandon des rizières.

Sur le plan socioéconomique, la féminisation de la filière associée au manque de moyens financier et technique constitue les maillons faibles de la production du riz dans cette partie du pays. La culture du riz est laissée aux femmes qui font face à des difficultés pour l'accès à la terre, le renouvellement de la main-d'œuvre et des ouvrages hydroagricoles. En revanche, la contribution des stratégies communautaires mises en place, laisse croire à une pérennisation et à un développement de la riziculture pluviale. Des perspectives de développement de la riziculture pluviale sont proposées. Parmi elles, l'augmentation et la diversification des revenus agricoles par la promotion d'une bonne politique de transformation, de commercialisation et de distribution. Il s'agit aussi de renforcer l'autonomie alimentaire en mettant en œuvre un programme d'appui, d'intensification et de modernisation de la riziculture de type familial qui se fonde sur des stratégies traditionnelles. Les résultats saillants révèlent que la riziculture pluviale est confrontée à des 
problèmes environnementaux et socioéconomiques qui freinent la productivité du riz.

\section{References:}

1. ANSD (2020). Situation économique et sociale du Sénégal 2017-2018. Ministère de l'économie, du plan et de la coopération, $17 \mathrm{p}$.

2. Atidegla C. S., Koumassi H. D., Mouzou E. T., \& Houssou E (2017). Variabilité climatique et production du riz dans le bas-fond de Dokomey au Benin. Journal de la Recherche Scientifique de l'Université de Lomé, pp. 259-276.

3. Diallo A. K. (2014). La riziculture pluviale de bas-fonds en Moyenne Casamance: Cas de la vallée de Badobar [Mémoire de Master en Géographie]. Université Cheikh Anta Diop de Dakar, 90 p.

4. Diédhiou S. O., Sy O., \& Margetic C. (2018). Impacts de la dynamique urbaine et de la variation hydrologique sur la sécurité alimentaire : Le cas du bassin versant de Djibélor (Ziguinchor). In Regards croisés sur les enjeux et perspectives environnementaux de la recomposition des espaces urbains et périurbains face aux changements climatiques. L'Harmattan Sénégal, pp. 267-281.

5. Diédhiou S. O., Sy O., \& Margetic C. (2019). La petite exploitation agricole familiale acteur de la sécurité alimentaire : Exemple de la vallée de Djibélor (Ziguinchor). Revue de géographie du laboratoire Leidi, pp. 160-173.

6. Faye C., Sy O., \& Diédhiou S. O. (2018). Analyse de phénomènes hydrologiques dans un bassin versant urbanisé et leur incidence sur l'agriculture: Cas de la ville de Ziguinchor (Sud du Sénégal). In Mbaye Ibrahima (dir.), la recomposition des espaces urbains et périurbains face aux changements climatiques en Afrique de l'Ouest. L’Harmattan Sénégal, pp. 177-194.

7. Kotchi J. K., Ouattara-Coulibaly Y. R., \& N'guessan G. K. (2018). Impact socio-économique de l'aménagement hydro-rizicole de Guiguidou dans la sous-préfecture de Divo (Côte d'Ivoire). EchoGéo, 43. https://doi.org/10.4000/echogeo.15275

8. Manzelli M., Fiorillo E., Bacci, M., \& Tarchiani V. (2015). Lowland rice production in southern Senegal (Middle Casamance) : Challenges and prospects for sustaining their restoration and development. Cahiers Agricultures, 24(5), pp. 301-312. https://doi.org/10.1684/agr.2015.0772

9. Mballo I., SY O., \& Faye C. (2019). Variabilité climatique et productions vivrières en Haute Casamance (Sud-Sénégal). Espace Géographique et Société Marocaine, CNRST-IMIST, pp. 161-178. 
10. Mendez del villar P. (2019). Comprendre les enjeux de l'agriculture: vers un renouveau de la riziculture pluviale au Sénégal. WillAgri. https://www.willagri.com/2019/06/24/vers-un-renouveau-de-lariziculture-pluviale-au-senegal/

11. Mendez del Villar P., \& Bauer J.-M. (2013). Rice in West Africa: Dynamics, policies and trends. Cahiers Agricultures, 22(5), pp. 336344. https://doi.org/10.1684/agr.2013.0657

12. Mendy V. (2018). Dégradation des agroécosystèmes et problématique de la revitalisation de la riziculture en Basse-Casamance [Thèse de doctorat de Géographie]. Université Assane SECK de Ziguinchor, 293 p.

13. Mendy V., \& Sy O. (2015). Riziculture en basse Casamance : Analyse des facteurs de crise et des stratégies populaires alternatives dans la commune d'Oulampane. Revue Espaces et Sociétés en Mutation, Numéro Spécial, pp. 146-165.

14. République du Sénégal (2014). Programme d'Accélération de la Cadence de l'agriculture sénégalaise (PRACAS). Ministère de l'Agriculture et de l'équipement rural, $112 \mathrm{p}$.

15. Sané T., SY O., Dièye E. H. B., Descroix L., \& Diaw A. T. (2015). De la pertinence des grands aménagements hydro-agricoles dans un contexte d'instabilité climatique: Le cas du barrage d'Affiniam en basse-Casamance. In Descroix Luc (ed.), Djiba S. (ed.), Sané T. (ed.), Tarchiani V. (ed.) Eaux et sociétés face au changement climatique dans le bassin de la Casamance: Actes de l'Atelier scientifique et du lancement de l'initiative "Casamance: Un réseau scientifique au service $d u$ développement en Casamance» (Fonds IRD [F A010069930]). L'Harmattan, pp. 117-134

16. Sané Tidiane (2017). Vulnérabilité et adaptabilité des systèmes agraires à la variabilité climatique et aux changements sociaux en Basse-Casamance [Thèse de doctorat en Géographie et Environnement]. Université Paris Diderot, 376 p.

17. Sow F. (1992). Femmes et tenures foncières au Sénégal [Rapport final]. CRDI/UCAD, $66 \mathrm{p}$.

18. Sy O., \& Mballo, I. (2017). Vulnérabilité et insécurité alimentaire dans le Bassin de l'Anambé : L'exploitation familiale à la croisée des chemins. FOLOFOLO, pp. 128-162.

19. Thior M., SY O., Sané T., Mballo I., Badiane A., \& Descroix L. (2019). Contraintes à la production rizicole et reconversion socioéconomique dans la commune de Diembering (Sénégal). Revue Ivoirienne de Géographie des Savanes, 6 juin, pp. 118-132.

20. Traoré, A., Traoré, K., Traoré, O., Bado, B. V., Nacro, B. H., \& Sedogo, M. P. (2015). Caractérisation des systèmes de production à 
base de riz pluvial strict dans les exploitations agricoles de la zone Sudsoudanienne du Burkina Faso. Int. J. Biol. Chem. Sci, Vol. 9 No. 6, pp. 2685-2697.

21. Vennetier, P. (1989). Centre, périphérie et flux intra-urbains dans les grandes villes d'Afrique noire. Annales de Géographie, 98(547), pp. 257-285. https://doi.org/10.3406/geo.1989.20906 